\title{
Is there a critical lesion site for unilateral spatial neglect? A meta-analysis using activation likelihood estimation
}

\author{
Pascal Molenberghs ${ }^{1}{ }^{*}$, Martin V. Sale ${ }^{2}$ and Jason B. Mattingley ${ }^{1,2}$ \\ School of Psychology, The University of Queensland, Brisbane, OLD, Australia \\ 2 Queensland Brain Institute, The University of Queensland, Brisbane, QLD, Australia
}

\author{
Edited by: \\ Konstantinos Priftis, University of \\ Padova, Italy \\ Reviewed by: \\ Radek Ptak, University of Geneva, \\ Switzerland \\ Paolo Bartolomeo, INSERM, France \\ *Correspondence: \\ Pascal Molenberghs, School of \\ Psychology, The University of \\ Queensland, McElwain Building, \\ St. Lucia, Brisbane, QLD 4072, \\ Australia. \\ e-mail:p.molenberghs@uq.edu.au
}

The critical lesion site responsible for the syndrome of unilateral spatial neglect has been debated for more than a decade. Here we performed an activation likelihood estimation (ALE) to provide for the first time an objective quantitative index of the consistency of lesion sites across anatomical group studies of spatial neglect. The analysis revealed several distinct regions in which damage has consistently been associated with spatial neglect symptoms. Lesioned clusters were located in several cortical and subcortical regions of the right hemisphere, including the middle and superior temporal gyrus, inferior parietal lobule, intraparietal sulcus, precuneus, middle occipital gyrus, caudate nucleus, and posterior insula, as well as in the white matter pathway corresponding to the posterior part of the superior longitudinal fasciculus. Further analyses suggested that separate lesion sites are associated with impairments in different behavioral tests, such as line bisection and target cancellation. Similarly, specific subcomponents of the heterogeneous neglect syndrome, such as extinction and allocentric and personal neglect, are associated with distinct lesion sites. Future progress in delineating the neuropathological correlates of spatial neglect will depend upon the development of more refined measures of perceptual and cognitive functions than those currently available in the clinical setting.

Keywords: unilateral spatial neglect, extinction, lesion mapping, ALE meta-analysis, line bisection, cancellation task

\section{INTRODUCTION}

Unilateral spatial neglect is typically defined as an inability to detect, attend or respond to stimuli in spatial locations contralateral to the side of cerebral damage (Heilman et al., 1993). The symptoms of spatial neglect are typically associated with cerebral damage involving the right hemisphere, although neglect also arises after left-sided lesions (Stone et al., 1993; Kleinman et al., 2007). Within the right hemisphere, neglect symptoms have been reported following damage to different brain regions, including the angular gyrus (Mort et al., 2003; Molenberghs and Sale, 2011), superior temporal cortex (Karnath et al., 2001, 2004), parahippocampus (Mort et al., 2003), temporo-parietal junction (Vallar and Perani, 1986; Karnath et al., 2003), inferior frontal lobe (Husain and Kennard, 1996; Rengachary et al., 2011), intraparietal sulcus (Molenberghs et al., 2008; Gillebert et al., 2011), insula (Karnath et al., 2004; Rengachary et al., 2011), putamen (Karnath et al., 2002, 2004), caudate nucleus (Karnath et al., 2002, 2004; Medina et al., 2009), pulvinar (Karnath et al., 2002), parieto-frontal cortex (Bartolomeo et al., 2007) and occipital lobe (Bird et al., 2006). Recent work also suggests that a common cause for spatial neglect is a disruption of white matter pathways connecting parietal and frontal areas (Doricchi and Tomaiuolo, 2003; Thiebaut de Schotten et al., 2005; Bartolomeo et al., 2007; Doricchi et al., 2008; Urbanski et al., 2008; Ptak and Schnider, 2010; Urbanski et al., 2011) and damage in a particular area could potentially cause functional changes well outside the critical lesion site (Corbetta et al., 2005; Hillis et al., 2005; He et al.,
2007; Corbetta and Shulman, 2011). It should be noted, however, that a focal lesion within a circumscribed brain area does not invariably alter cerebral perfusion in functionally relevant, but structurally unaffected, regions (Zopf et al., 2009) and some authors have argued that gray matter damage is the most common cause of neglect (Karnath et al., 2009). Yet this latter study was based on white matter damage on the Jülich brain atlas (Bürgel et al., 2006), which is built on coronal anatomical slices of post-mortem brains, and as a consequence could underestimate the extension of caudo-rostral pathways such as the superior longitudinal fasciculus.

Different lesion sites have been associated with different aspects of the neglect syndrome and the condition is now widely accepted as a heterogeneous syndrome (Stone et al., 1998; Hillis, 2006; Karnath and Rorden, in press). For example, patients with visual extinction, but without neglect, can detect single stimuli presented briefly and in isolation in either hemispace, but fail to detect the more contralesional event when two stimuli occur simultaneously on both sides (Karnath et al., 2003). Other subdivisions in the neglect syndrome have been made. Personal neglect, for example, refers to cases in which the patient is unaware of the contralesional side of the body (Committeri et al., 2007; Baas et al., 2011). By contrast, extrapersonal neglect refers to patients who ignore the contralesional side of the external environment beyond the body, either within or beyond reaching space (Committeri et al., 2007). Allocentric neglect concerns a failure to perceive the contralesional side of individual objects 
(e.g., items in a complex scene or words) regardless of their orientation or position relative to the body (Medina et al., 2009; Chechlacz et al., 2010). By contrast, egocentric neglect refers to a deficit in perceiving stimuli located on the contralesional side of space relative to the body midline (Chechlacz et al., 2010).

In order to probe these different symptoms of the neglect syndrome, a wide variety of clinical tests have been developed (Karnath et al., 2003; Rorden et al., 2006; Grimsen et al., 2008; Marsh and Hillis, 2008; Verdon et al., 2010), the majority of which test for neglect within the visual modality. Most common among these are cancellation tasks, in which patients use a pen to mark individual targets scattered on a page (Albert, 1973; Gauthier et al., 1989; Ota et al., 2001), and line bisection tasks in which patients are required to indicate the midpoint of several horizontal lines (Schenkenberg et al., 1980; Wilson et al., 1987; Halligan et al., 1990). These tests were designed to be easy to administer and score, but lack specificity in terms of the underlying perceptual and cognitive deficits they measure (Vandenberghe and Gillebert, 2009; Verdon et al., 2010). Some consideration of the underlying properties of these clinical tests is important when attempting to determine whether there is a critical brain region whose damage most commonly causes symptoms of neglect. We addressed this issue by undertaking anatomical analyses separately for lesion maps obtained from studies that employed either cancellation or line bisection tasks to define neglect.

To date, the published group studies that have investigated the anatomy of neglect have been limited by small sample sizes and wide variability in lesion sites. The principal motivation for the present study was thus to add clarity to the information presently available by determining for the first time which, if any, lesion sites are consistently associated with the neglect syndrome across different studies. Our main goal was to summarize the data currently available and do this in an unbiased way. Therefore, we performed an activation likelihood estimation (ALE) metaanalysis (Laird et al., 2005; Eickhoff et al., 2009), which has been used previously as an objective measure to quantify the relationship between brain anomalies and different syndromes (Glahn et al., 2008; Rotge et al., 2010; Ferreira et al., 2011).

Since spatial neglect is often used as a broad term to describe different behavioral deficits in clinical settings and research studies, we first identified all published group studies of neglect in which lesions had been mapped and that reported the peak coordinates of the critical lesion site. We also performed an additional qualitative analysis by subdividing the peak coordinates from different subtests such as target cancellation and line bisection. Likewise, we examined peak coordinates for subgroups of neglectlike symptoms such as extinction, personal, extrapersonal, egocentric and allocentric neglect and provide them in an objective framework. The findings, which imply an objective and coherent network of brain areas associated with different neglect measures and symptoms, should prove useful for researchers and clinicians involved in the management and rehabilitation of patients with this debilitating neurological condition. They should also assist experts in the field to develop more refined measures of perceptual and cognitive functions associated with the spatial neglect syndrome in the future.

\section{MATERIALS AND METHODS LITERATURE SELECTION AND EXCLUSION CRITERIA}

We searched the Web of Science database (http://apps. isiknowledge.com) in January 2012 using the keywords "spatial neglect," "lesion mapping," "extinction," “inattention," "hemineglect," "hemispatial neglect," and "unilateral neglect". The inclusion criteria for our analyses were as follows:

1. Studies that dealt with spatial neglect in human patients were included, whereas those that did not were excluded (e.g., the search also uncovered studies focused on lesions in nonhuman species, as well as review articles).

2. Studies that used lesion localization were included, whereas those that employed other techniques (e.g., behavioral tests, positron emission tomography (PET) or perfusion studies) were excluded.

3. Because we were only interested in consistent lesion sites across patient groups, we only included studies that performed group lesion-overlap analyses; other studies (e.g., single case studies) were excluded.

4. Studies that did not report the coordinates of damage or for which the coordinates could not be obtained through personal communication were excluded.

A total of 20 studies that were found in the Web of Science database or that were known to the authors matched all the inclusion criteria and were thus entered into the meta-analysis (see Table 1 for a complete list of the included studies).

\section{SELECTION OF PEAK COORDINATES}

From the 20 studies that passed the inclusion criteria listed above, we extracted the peak coordinates reported on the basis of the authors' lesion mapping analyses. To minimize overrepresentation by one particular region based on a single study, we only included peak coordinates that were separated by more than $10 \mathrm{~mm}$ from each other in $x, y$, and $z$ space in the same study and for the same test. Additionally, if the voxels reported in the original study were reported in Talairach space we transformed them into Montreal Neurological Institute (MNI) space using the icbm2tal algorithm (Lancaster et al., 2007) implemented in the Ginger ALE software (Eickhoff et al., 2009). In total, 69 foci were included in the overall analysis (see Table 1 and Figure 1 for details).

\section{ACTIVATION LIKELIHOOD ESTIMATION (ALE)}

To identify the brain regions consistently lesioned in the included studies, we performed an ALE analysis (Eickhoff et al., 2009). Although ALE analysis was originally developed to quantify consistent activation patterns in neuroimaging studies, it has also been used in meta-analyses of brain anomalies in such syndromes as obsessive-compulsive disorder (Rotge et al., 2010), schizophrenia (Glahn et al., 2008; Cheung et al., 2010), Alzheimer's disease (Ferreira et al., 2011) and autistic spectrum disorders (Cheung et al., 2010). We used Version 2.0, which has advantages over earlier ALE algorithms (Turkeltaub et al., 2002; Laird et al., 2005) in that rather than testing for an above-chance clustering between activated foci, it assesses above-chance clustering of activated foci 
Table 1 | Overview of the 20 studies and peak coordinates used in the ALE analysis.

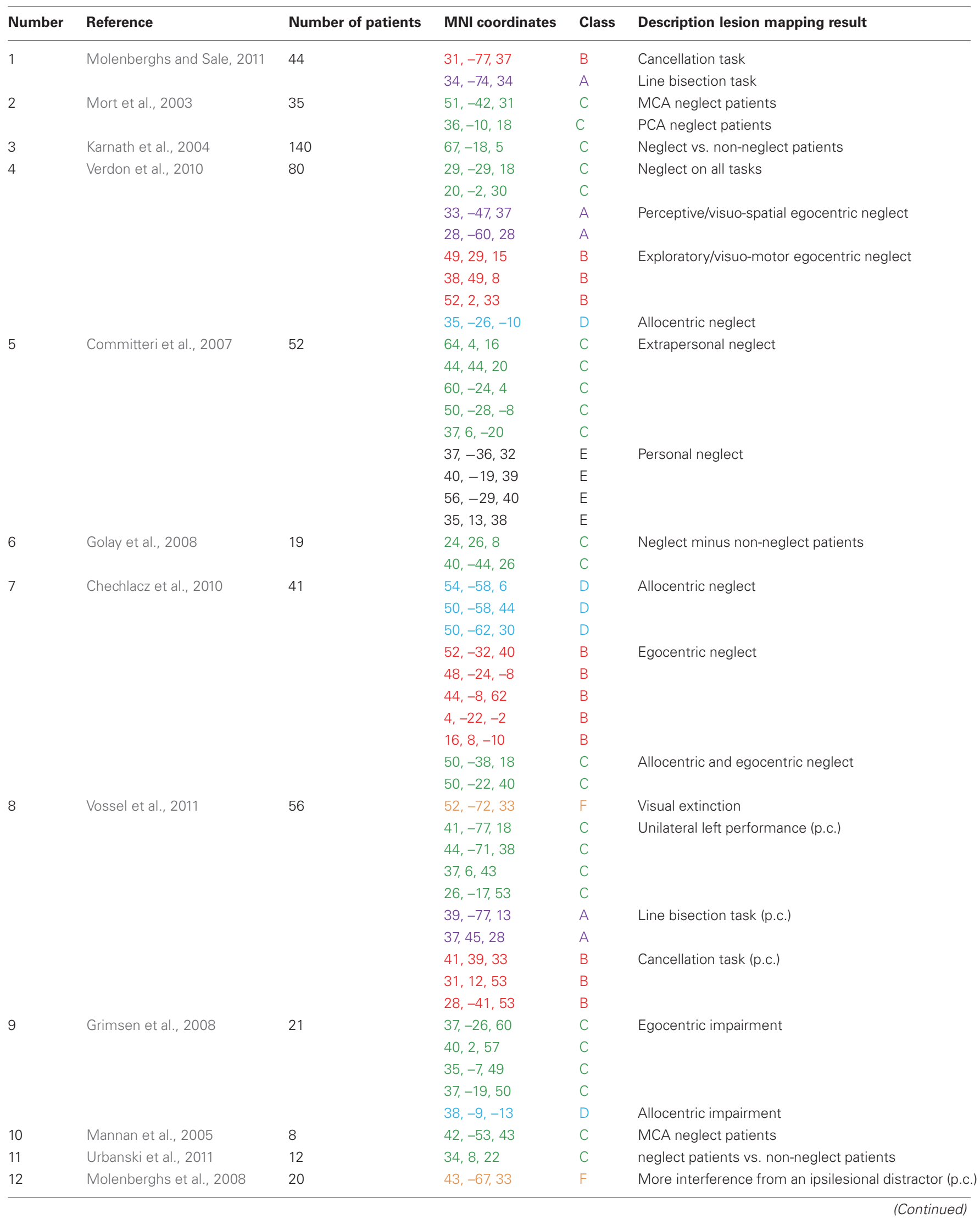


Table 1 | Continued

\begin{tabular}{|c|c|c|c|c|c|}
\hline Number & Reference & Number of patients & MNI coordinates & Class & Description lesion mapping result \\
\hline \multirow[t]{3}{*}{14} & Karnath et al., 2003 & 27 & $69,-9,0$ & C & Neglect \\
\hline & & & $63,-55,27$ & $\mathrm{~F}$ & \\
\hline & & & $67,-49,8$ & $\mathrm{~F}$ & \\
\hline & & & $29,-38,53$ & C & \\
\hline \multirow[t]{2}{*}{16} & Doricchi and Tomaiuolo, 2003 & 21 & $39,-8,26$ & C & All neglect patients minus controls \\
\hline & & & $30,-5,35$ & C & \\
\hline 17 & Baas et al., 2011 & 22 & $47,-42,20$ & $E$ & Patients with minus without personal neglect \\
\hline 18 & Ptak and Schnider, 2010 & 29 & $30,-22,22$ & C & Neglect patients minus control patients \\
\hline 20 & & & $55,-21,13$ & C & Viewer-centered neglect (p.c.) \\
\hline
\end{tabular}

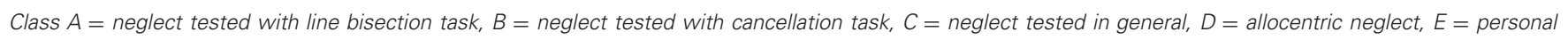
neglect and $F=$ spatial extinction. p.c. $=$ coordinates obtained through personal communication.

between experiments, thus permitting random-effects inference. The ALE analysis was conducted using the standard settings in the Ginger ALE software (Eickhoff et al., 2009). The test was corrected for multiple comparisons using the false discovery rate (FDR) method with $p<0.05$, and a suggested minimum volume of $200 \mathrm{~mm}^{3}$ voxels was used to define a cluster. The maps of the ALE values were superimposed on a ch2better.nii.gz atlas using MRIcron software (http://www.mricro.com/mricron).

\section{SUBDIVISIONS}

As noted in the Introduction, spatial neglect is increasingly considered a heterogeneous syndrome (Stone et al., 1998; Verdon et al., 2010; Karnath and Rorden, in press). Distinctions have been drawn between spatial neglect and extinction (Ogden, 1985; Liu et al., 1992; Di Pellegrino and De Renzi, 1995; Cocchini et al., 1999; Karnath et al., 2003), personal and extrapersonal neglect (Guariglia and Antonucci, 1992; Beschin and Robertson, 1997; Committeri et al., 2007; Baas et al., 2011), and egocentric and allocentric neglect (Hillis et al., 2005; Grimsen et al., 2008; Marsh and Hillis, 2008; Medina et al., 2009; Chechlacz et al., 2010). Moreover, it has been suggested that different tests for visual neglect, such as cancellation and line bisection, are associated with different lesion sites (Karnath et al., 2004; Rorden et al., 2006; Verdon et al., 2010). Therefore, in addition to the main analysis we subdivided the coordinates into six subclasses (See Table 1 and Figures 1 and $\mathbf{3}$ for details). Subclass A included all coordinates in which neglect was specifically tested for using a line bisection task (purple spheres); Subclass B included all coordinates in which neglect was specifically tested for using a cancellation task (red spheres); Subclass C was used for coordinates derived from studies in which several different tasks (e.g., target cancellation, line bisection, copying, clinical observation) were used in combination to test for the presence of neglect (green spheres);
Subclass D included coordinates for allocentric neglect (blue spheres); Subclass E included coordinates for personal neglect (black spheres); and Subclass F included coordinates for spatial extinction (orange spheres). Since dividing the data into separate subclasses did not provide enough coordinates to justify separate ALE meta-analyses, we plotted the individual coordinates on an inflated cortical surface to give a qualitative overview of the anatomy derived from the different studies.

\section{RESULTS AND DISCUSSION META-ANALYSIS ACROSS ALL INCLUDED STUDIES}

The ALE meta-analysis of all included studies (Table 1 ) revealed nine significant clusters in total (See Figure 2 and Table 2 for details), all of which were located in the right hemisphere. The largest area of damage consistently associated with neglect included white matter corresponding to the posterior part of the superior longitudinal fasciculus (Mori et al., 2005). Other significant clusters were located in: (1) An area located at the border region between the posterior middle temporal gyrus and angular gyrus; (2) The inferior parietal lobule; (3) The caudate nucleus; (4) An area located at the border between the horizontal segment of the intraparietal sulcus and postcentral sulcus; (5) The precuneus; (6) An area including the superior temporal gyrus and superior temporal sulcus; (7) The posterior insula; and (8) The middle occipital gyrus.

\section{SUBDIVISIONS}

\section{Line bisection vs. target cancellation}

Our analysis shows that most of the lesions associated with line bisection deficits (purple spheres in Figure 3A) are located more posteriorly than those associated with target cancellation deficits (red spheres in Figure 3B), although one set of coordinates for line bisection, from the study by Vossel et al. (2011), 


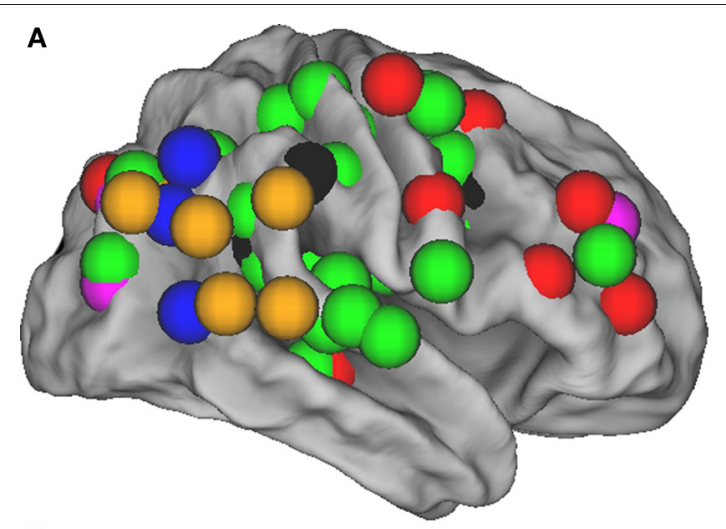

B

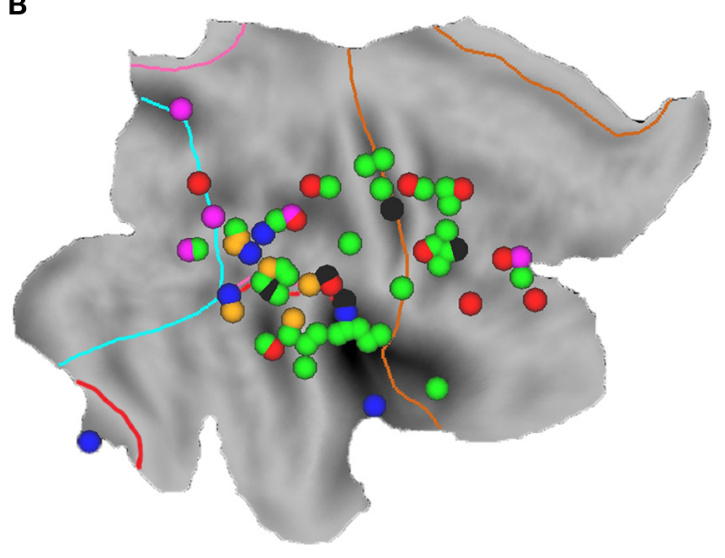

FIGURE 1 | Overview of all regions associated with unilateral spatial neglect, based upon 20 lesion mapping studies (see Table 1), superimposed on the cortical surface of the right hemisphere using CARET software (v5.64 http://brainmap.wustl.edu/caret.html).

(A) Fiducial map. Purple spheres $=$ neglect tested with line bisection tasks; red spheres $=$ neglect tested with cancellation tasks; green spheres $=$ neglect tested with a combination of tasks; blue spheres = allocentric neglect; black spheres $=$ personal neglect; orange spheres $=$ spatial extinction. (B) Flat map with identical spheres as in A. Cyan line indicates the occipital lobe border; pink line $=$ parietal lobe; red line $=$ temporal lobe; brown line $=$ frontal lobe; regions outside borders $=$ limbic lobe.

is located in the right middle frontal gyrus. The coordinates associated with neglect on target cancellation are quite widely distributed over dorsolateral prefrontal and parietal areas. It has been suggested that poor performance on the line bisection task is associated with more posterior lesions (Rorden et al., 2006; Verdon et al., 2010) because line bisection involves a more "perceptual or representational" deficit, whereas target cancellation deficits could also result from problems with "motor exploration" (Binder et al., 1992). This explanation is consistent with our finding of more frontal foci across studies that used target cancellation to assess neglect (Figure 3B). On the other hand, a recent study (Molenberghs and Sale, 2011) has shown that performance on line bisection and target cancellation in an unbiased clinical sample of left and right hemisphere stroke patients was highly correlated $(r=0.76)$, and that both tasks are associated with lesions in the right posterior angular gyrus. In more homogeneous (i.e., pre-selected) patient groups these correlations seem to be less pronounced (Binder et al., 1992; Ferber and Karnath, 2001), probably because of the reduced variance in the behavioral data. Another explanation for the discrepancy could be a difference in the approach to administering the line bisection task. For example, in the study by Ferber and Karnath (2001), the lines had been placed at the rightmost part of the sheet, which likely resulted in reduced or absent deviations from the midline in neglect patients (Schenkenberg et al., 1980).

\section{Allocentric vs. egocentric neglect}

Four of the studies that met our inclusion criteria (Grimsen et al., 2008; Medina et al., 2009; Chechlacz et al., 2010; Verdon et al., 2010) specifically examined allocentric neglect. Grimsen et al. (2008) found that allocentric neglect was associated with ventral lesions involving areas including the parahippocampal gyrus, whereas egocentric neglect was associated with more dorsal lesions in the premotor cortex. A similar dorsal versus ventral distinction between ego- and allocentric neglect was also found in the study by Medina et al. (2009). The parahippocampal gyrus was also implicated as the critical lesion site for allocentric neglect in a study by Verdon et al. (2010). In addition, these authors found that the critical lesion site for allocentric neglect extended into the middle temporal gyrus, as did Chechlacz et al. (2010), although in the latter study the damaged area also extended into posterior regions including the posterior temporal and angular gyrus (Figure 3D).

\section{Personal vs. extrapersonal neglect}

Personal neglect has been associated with more dorsal lesions than observed in patients with extrapersonal neglect (Committeri et al., 2007). These regions, which are shown in Figure 3E, are thought to be involved in coding proprioceptive body information such as the somatosensory cortex and more abstract body information such as the supramarginal gyrus (Committeri et al., 2007) and temporo-parietal junction (Baas et al., 2011).

\section{Spatial neglect vs. spatial extinction}

All studies that included a measure of extinction yielded lesions lying within posterior cortical regions, including the angular gyrus (Karnath et al., 2003; Molenberghs et al., 2008; Vossel et al., 2011) and temporo-parietal junction (Karnath et al., 2003). This is consistent with the view that spatial extinction is associated with more posterior parietal regions subserving stimulus competition (Karnath et al., 2003; Molenberghs et al., 2008; Gillebert et al., 2011), whereas the spatial neglect syndrome which is usually measured with a wider variety of behavioral measures is associated with multiple lesion sites. We note, however, that some previous studies have suggested that extinction arises as a nonspecific consequence of any unilateral lesion, perhaps reflecting a general competitive imbalance in sensory or other cortical areas (Birch et al., 1967; Farah et al., 1991; Vallar et al., 1994; Duncan et al., 1997). While this may be true to some extent, we note that these conclusions were based on clinical investigations that lacked high-resolution MRI data and statistical analytic techniques, such as voxel-based lesion-symptom mapping (Bates et al., 2003; Rorden et al., 2007), which can uncover subtle but consistent lesion foci across patients. 


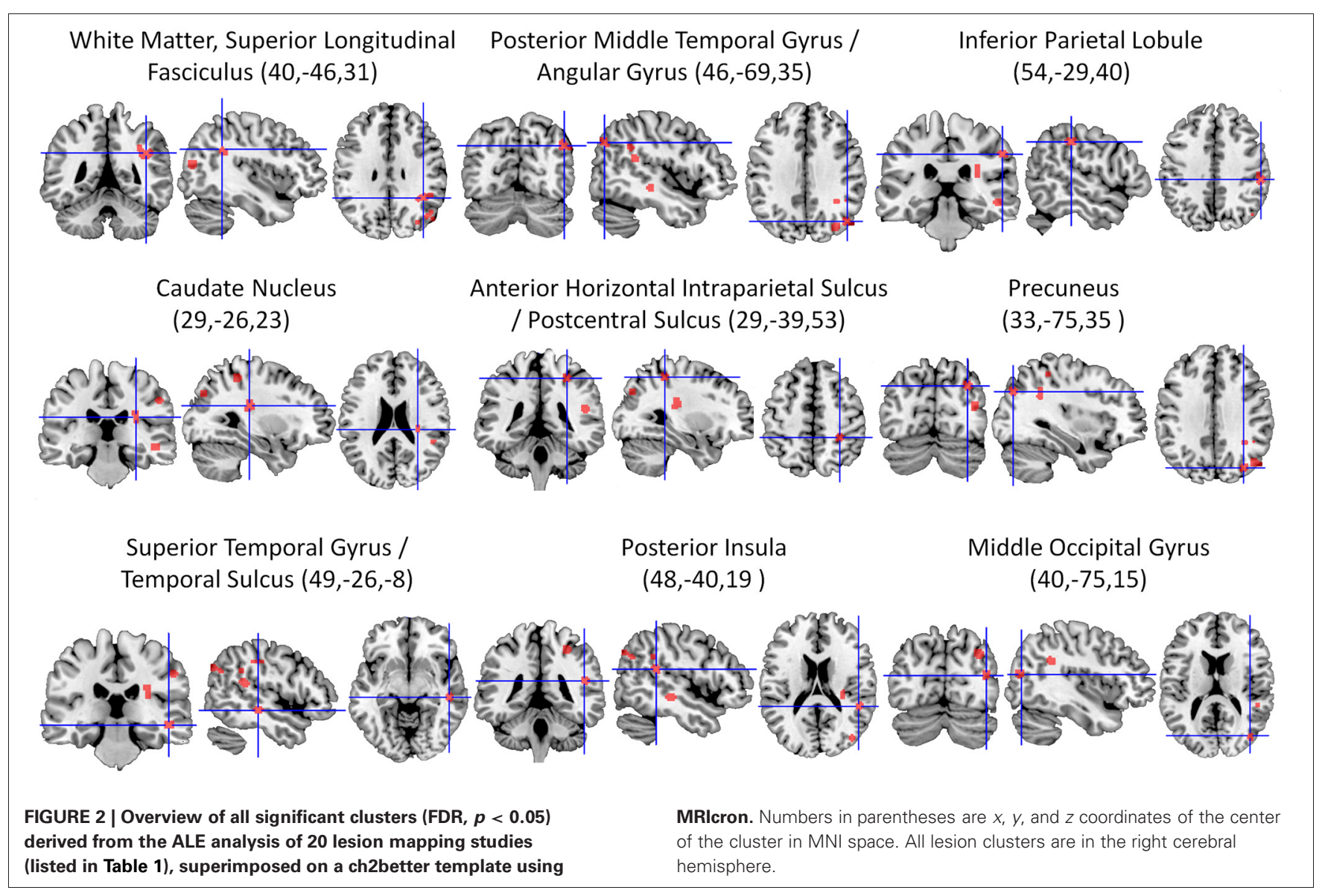

Table 2 | Significant clusters (FDR, $\boldsymbol{p}<\mathbf{0 . 0 5}$ ) revealed by the ALE analysis of the 20 lesion mapping studies.

\begin{tabular}{llll}
\hline Cluster & Cluster size in $\mathbf{~ m m}^{\mathbf{3}}$ & $\begin{array}{l}\text { Center MNI coordinates } \\
(\boldsymbol{x}, \boldsymbol{y}, \boldsymbol{z})\end{array}$ & Anatomical region \\
\hline 1 & 704 & $40,-46,31$ & White matter (right superior longitudinal fasciculus) \\
2 & 448 & $46,-69,35$ & Right posterior middle temporal gyrus/right angular gyrus \\
3 & 376 & $54,-29,40$ & Right inferior parietal lobule \\
4 & 352 & $29,-26,23$ & Right caudate nucleus \\
5 & 336 & $29,-39,53$ & Right anterior horizontal intraparietal sulcus /postcentral sulcus \\
6 & 320 & $33,-75,35$ & Right precuneus \\
7 & 288 & $49,-26,-8$ & Right superior temporal gyrus / right superior temporal sulcus \\
8 & 256 & $48,-40,19$ & Right posterior insula \\
9 & 240 & $40,-75,15$ & Right middle occipital gyrus \\
\hline
\end{tabular}

\section{GENERAL DISCUSSION \\ COMMON REGIONS ASSOCIATED WITH SPATIAL NEGLECT}

From the wide variety of brain areas associated with unilateral spatial neglect (Figure 1), nine regions emerged consistently across the studies included in our meta-analysis (Table 2 and Figure 2). These included regions typically associated with spatial neglect, such as the posterior temporal cortex (Karnath et al., 2004) and inferior parietal lobule (Mort et al., 2003), but also other regions less commonly associated with spatial neglect such as the occipital lobe. Although neglect is less common after posterior cerebral artery (PCA) infarction than after middle cerebral artery (MCA) infarction (Mort et al., 2003), it is not uncommon for PCA patients with occipital lobe lesions to suffer from neglect, probably because of damage to white matter pathways connecting the parahippocampal and angular gyrus (Bird et al., 2006). This probably explains why the right middle occipital gyrus was one of the critical lesion sites identified in our meta-analysis. Specific gray matter structures are more localized than long-range white matter pathways, and different sectors along a long-range white matter pathway can 


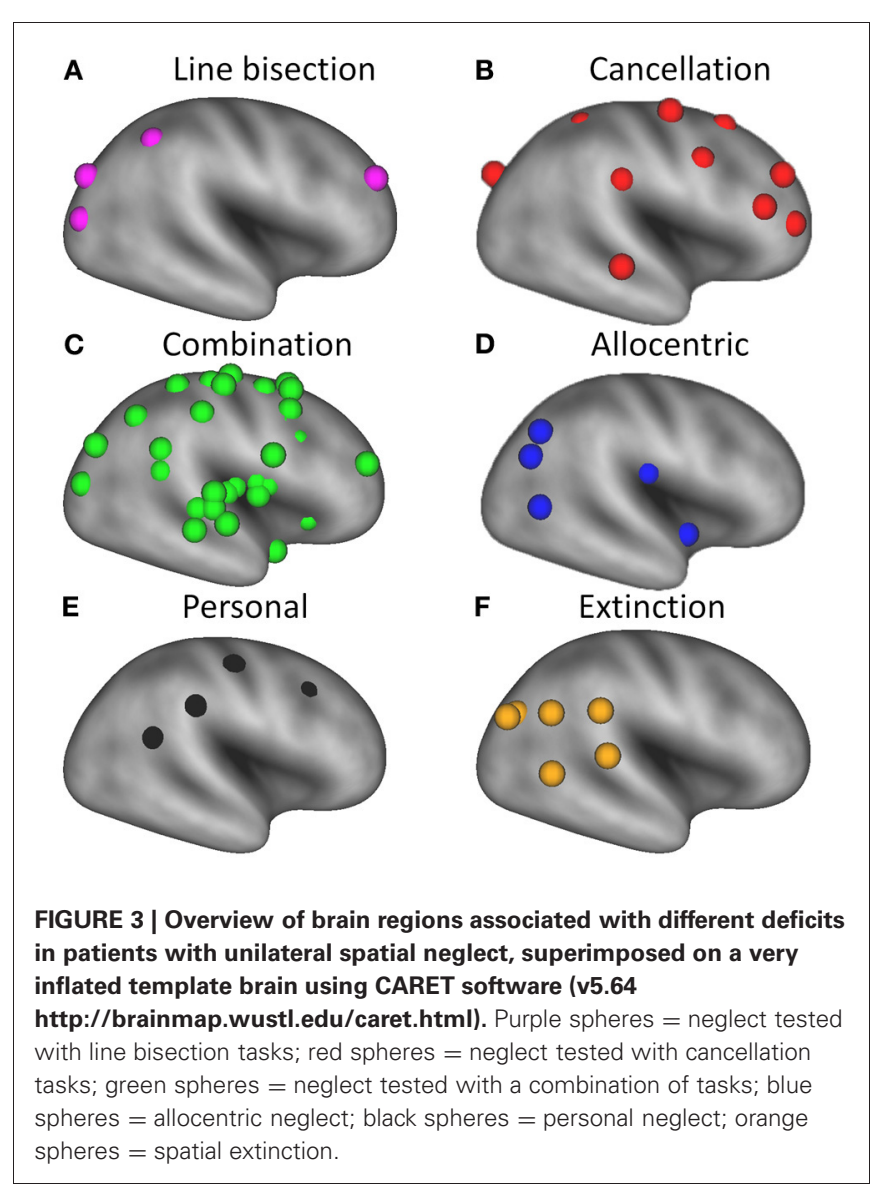

produce similar effects by disconnecting the fascicle, independent of the precise location of the interruption (Catani and Mesulam, 2008). It is possible, therefore, that our meta-analytic method under-represents the contribution of white matter lesions to spatial neglect. Nevertheless, consistent with the view that neglect can arise from white matter lesions connecting parietal and frontal areas (Doricchi and Tomaiuolo, 2003; Bartolomeo et al., 2007; Doricchi et al., 2008; Ptak and Schnider, 2010), our meta-analysis revealed that the largest region involved in the development of spatial neglect was a white matter lesion corresponding to the superior longitudinal fasciculus (Mori et al., 2005). Most of the significant regions in our analysis form the core of a previously described, "circuit-breaking" ventral frontoparietal attention network (Corbetta and Shulman, 2002; Corbetta et al., 2008), and lesions in this area can also lead to functional changes in the dorsal frontoparietal selective attention network (Corbetta et al., 2005; He et al., 2007; Corbetta and Shulman, 2011). Given the fact that most of these regions are situated around the center of the vascularization territory of the MCA it is no surprise that they emerged consistently and reliably from our meta-analysis. Vascular brain damage normally respects arterial territories and therefore will inevitably involve some brain regions more than others. This consideration should be borne in mind when interpreting the results of any lesion-based analysis, and our ALE approach is no exception. More posterior parietal regions that form the core components of this dorsal network, such as the intraparietal sulcus and superior parietal lobule, which are typically activated in neuroimaging studies on selective attention (Corbetta and Shulman, 2002; Woldorff et al., 2004; Molenberghs et al., 2007; Serences and Yantis, 2007; Molenberghs et al., 2008; Vandenberghe and Gillebert, 2009; Vandenberghe et al., in press), are less likely to be affected by stroke because they are situated at the border of the MCA and therefore have a smaller chance of being affected by stroke than the more central regions (Tatu et al., 2001). Recent evidence (Gillebert et al., 2011; Vandenberghe et al., in press), however, suggests that small focal lesions restricted to these regions can cause the same neglect-like symptoms as typically found in patients with more ventral damage.

\section{NEGLECT AS A HETEROGENEOUS SYNDROME}

The term "unilateral spatial neglect" is used to describe a range of functional impairments, and the condition has increasingly become viewed as heterogeneous (Stone et al., 1998; Verdon et al., 2010; Karnath and Rorden, in press). This view is supported by the current meta-analysis, which shows that symptoms of the neglect syndrome considered together are associated with a widely distributed matrix of brain regions (Figure 1). A more detailed analysis of the different subclasses of neglect symptoms, such as visual extinction, allocentric, egocentric, personal and extrapersonal neglect, is important for characterizing the neural circuits that underlie these dissociable functional deficits (Figure 3). Further clues to this underlying circuitry can be gleaned from neuroimaging studies of neurologically healthy participants as they undertake conventional neglect-type tasks. For example, brain imaging studies of line bisection have implicated posterior cortical regions including the inferior and superior parietal lobule (Fink et al., 2000). Likewise, imaging studies of visual search, a task which in some respects at least resembles target cancellation, have shown that activity in the superior frontal sulcus is associated with effortful, conscious visual search (Leonards et al., 2000). These findings in healthy participants are consistent with data from our lesion meta-analysis, which showed that deficits on cancellation tasks tend to be associated with more anterior lesions (Figure 3B), whereas those with deficits on line bisection were associated with more posterior lesions (Figure 3A).

\section{DIFFERENT CRITERIA, TESTS AND TECHNIQUES PRODUCE DIFFERENT LESION PATTERNS}

The 20 studies included in the meta-analysis used different criteria to identify neglect in their patient samples. For example, in the study by Mort et al. (2003) the criterion for classifying a patient as having neglect on line bisection was a 3 percent rightward deviation from the midline, whereas in the study of Molenberghs and Sale (2011) the criterion was 9.5 percent. It follows that a given patient identified as having neglect in one study might not be classified as such in another. It will therefore be critical in future studies for investigators to use a continuous behavioral score in lesion mapping analyses, so that the severity of symptoms can be taken into account. In previous studies (Karnath et al., 2001, 2003; Mort et al., 2003; Rorden et al., 2006) patients were divided into dichotomous groups according to arbitrary 
cut-offs, but recent developments in lesion mapping approaches (Rorden et al., 2007), and the inclusion of continuous behavioral scores as variables (Molenberghs et al., 2008; Verdon et al., 2010; Karnath et al., 2011; Molenberghs and Sale, 2011; Vossel et al., 2011) has improved the inferences that can be drawn from such studies. In addition, the time of testing post stroke (acute vs. chronic stage) is also an important factor that differs between studies and this can result in different lesion sites (Karnath et al., 2011).

The studies included in our meta-analysis also varied widely in the actual tests administered to assess neglect. Across the 20 studies there were seven different cancellation tasks [Line crossing (Albert, 1973), Ota's search task (Ota et al., 2001), Apple Cancellation Task (Chechlacz et al., 2010), Letter Cancellation Test (Weintraub and Mesulam, 1985), Star Cancellation Test (Halligan et al., 1989), cancellation tests from the BIT (Wilson et al., 1987), Bells Test (Gauthier et al., 1989) and Mesulam shape cancellation task (Mesulam, 1985)] and five different line bisection tasks [110 lines of varying length (Halligan et al., 1990), 18 lines of varying length (Schenkenberg et al., 1980), 8 lines of varying length (Urbanski et al., 2011), 10 lines of equal length (Ferber and Karnath, 2001), and three lines of equal length from the Behavioural Inattention Test (Wilson et al., 1987)]. These differences might well have contributed to inconsistencies in critical lesion sites reported across studies. Thus, for example, the targets in the Bells test (Gauthier et al., 1989) are small and densely interspersed amongst many distractor items, whereas the line crossing test (Albert, 1973) consists of sparsely distributed line segments with no visual distractors.

In addition to different behavioral measures, the studies employed different neuroimaging techniques. For example, some included low-resolution CT scans from which lesions were drawn manually onto standard templates (Karnath et al., 2004), while

\section{REFERENCES}

Albert, M. L. (1973). A simple test of visual neglect. Neurology 23, 658-664.

Baas, U., de Haan, B., Graessli, T., Karnath, H.-O., Mueri, R., Perrig, W. J., Wurtz, P., and Gutbrod, K. (2011). Personal neglect-a disorder of body representation? Neuropsychologia 49, 898-905.

Bartolomeo, P., Thiebaut de Schotten, M., and Doricchi, F. (2007). Left unilateral neglect as a disconnection syndrome. Cereb. Cortex 17, 2479-2490.

Bates, E., Wilson, S. M., Saygin, A. P., Dick, F., Sereno, M. I., Knight, R. T., and Dronkers, N. F. (2003). Voxel-based lesion-symptom mapping. Nat. Neurosi. 6, 448-450.

Bays, P. M., Singh-Curry, V., Gorgoraptis, N., Driver, J., and Husain, M. (2010). Integration of goal- and stimulus-related visual signals revealed by damage to

others used high-resolution MRI scans in which the lesions were mapped directly onto the original image (Mort et al., 2003; Molenberghs et al., 2008).

\section{THE WAY FORWARD}

In addition to revealing the critical lesion sites associated with the various clinical manifestations of visual neglect, a key message of the current investigation is that there is a need to develop more sensitive and nuanced assessment tools to characterize the different facets of this heterogeneous syndrome. For example, a typical test for spatial neglect, such as target cancellation, involves both visuo-spatial and visuo-motor components. Impairment in either domain could therefore result in the same, abnormal score on the test, but due to deficits in different underlying functional processes. It will be important to bring laboratory tests into the clinic in an effort to identify specific cognitive functions by examining each in isolation [e.g., selective visual attention (Corbetta et al., 2005; Molenberghs et al., 2008; Bays et al., 2010; Gillebert et al., 2011; Vossel et al., 2011)]. Combining more specific descriptions of the neglect syndrome with better clinical measures that isolate specific cognitive functions should yield more consistent lesion mapping results in the future.

\section{ACKNOWLEDGMENTS}

This work was supported by a University of Queensland Postdoctoral Fellowship awarded to Pascal Molenberghs, a National Health and Medical Research Council (NHMRC) Postdoctoral Training Fellowship awarded to Martin V. Sale, and an NHMRC Project Grant awarded to Jason B. Mattingley and Pascal Molenberghs. We would like to thank Jared Medina, Mikolaj Pawlak, Jennifer Rengachary, Simone Vossel, Philipp Eschenbeck and Tim Shallice for kindly providing us with the coordinates from their papers.

tracts of the human brain: threedimensional mapping at microscopic resolution, topography and intersubject variability. Neuroimage 29, 1092-1105.

Catani, M., and Mesulam, M. (2008). The arcuate fasciculus and the disconnection theme in language and aphasia: history and current state. Cortex 44, 953-961.

Chechlacz, M., Rotshtein, P., Bickerton, W.-L., Hansen, P. C., Deb, S., and Humphreys, G. W. (2010). Separating neural correlates of allocentric and egocentric neglect: distinct cortical sites and common white matter disconnections. Cogn. Neuropsychol. 27, 277-303.

Cheung, C., Yu, K., Fung, G., Leung, M., Wong, C., Li, Q., Sham, P., Chua, S., and Mcalonan, G. (2010). Autistic disorders and schizophrenia: related or remote? An anatomical likelihood estimation. Plos One 5:12233. doi: 10.1371/journal.pone.0012233
Cocchini, G., Cubelli, R., Della Sala, S., and Beschin, N. (1999). Neglect without extinction. Cortex 35, 285-313.

Committeri, G., Pitzalis, S., Galati, G., Patria, F., Pelle, G., Sabatini, U., Castriota-Scanderbeg, A., Piccardi, L., Guariglia, C., and Pizzamiglio, L. (2007). Neural bases of personal and extrapersonal neglect in humans. Brain 130, 431-441.

Corbetta, M., Kincade, M. J., Lewis, C., Snyder, A. Z., and Sapir, A. (2005). Neural basis and recovery of spatial attention deficits in spatial neglect. Nat. Neurosci. 8, 1603-1610.

Corbetta, M., Patel, G., and Shulman, G. L. (2008). The reorienting system of the human brain: from environment to theory of mind. Neuron 58, 306-324.

Corbetta, M., and Shulman, G. L. (2002). Control of goal-directed and stimulus-driven attention in the brain. Nat. Rev. Neurosci. 3, 201-215. 
Corbetta, M., and Shulman, G. L. (2011). Spatial neglect and attention networks. Annu. Rev. Neurosci. 34, 569-599.

Di Pellegrino, G., and De Renzi, E. (1995). An experimental investigation on the nature of extinction. Neuropsychologia 33, 153-170.

Doricchi, F., Thiebaut de Schotten, M., Tomaiuolo, F., and Bartolomeo, P. (2008). White matter (dis)connections and gray matter (dys) functions in visual neglect: gaining insights into the brain networks of spatial awareness. Cortex 44, 983-995.

Doricchi, F., and Tomaiuolo, F. (2003). The anatomy of neglect without hemianopia: a key role for parietalfrontal disconnection? Neuroreport 14, 2239-2243.

Duncan, J., Humphreys, G., and Ward, R. (1997). Competitive brain activity in visual attention. Curr. Opin. Neurobiol. 7, 255-261.

Eickhoff, S. B., Laird, A. R., Grefkes, C., Wang, L. E., Zilles, K., and Fox, P. T. (2009). Coordinate-based activation likelihood estimation meta-analysis of neuroimaging data: a random-effects approach based on empirical estimates of spatial uncertainty. Hum. Brain Mapp. 30, 2907-2926.

Eschenbeck, P., Vossel, S., Weiss, P. H., Saliger, J., Karbe, H., and Fink, G. R. (2010). Testing for neglect in righthemispheric stroke patients using a new assessment battery based upon standardized activities of daily living (ADL). Neuropsychologia 48, 3488-3496.

Farah, M. J., Monheit, M. A., and Wallace, M. A. (1991). Unconscious perception of extinguished visual stimuli: reassessing the evidence. Neuropsychologia 29, 949-958.

Ferber, S., and Karnath, H. O. (2001). How to assess spatial neglect - line bisection or cancellation tasks? J. Clin. Exp. Neuropsychol. 23, 599-607.

Ferreira, L. K., Diniz, B. S., Forlenza, O. V., Busatto, G. F., and Zanetti, M. V. (2011). Neurostructural predictors of Alzheimer's disease: a metaanalysis of VBM studies. Neurobiol. Aging 32, 1733-1741.

Fink, G. R., Marshall, J. C., Shah, N. J., Weiss, P. H., Halligan, P. W., Grosse-Ruyken, M., Ziemons, K., Zilles, K., and Freund, H. J. (2000). Line bisection judgments implicate right parietal cortex and cerebellum as assessed by fMRI. Neurology 54, 1324-1331.

Gauthier, L., Dehaut, F., and Joanette, Y. (1989). The Bells Test - A quantitative and qualitative test for visual neglect. Int. J. Clin. Neuropsychol. 11, 49-54.

Gillebert, C. R., Mantini, D., Thijs, V., Sunaert, S., Dupont, P., and Vandenberghe, R. (2011). Lesion evidence for the critical role of the intraparietal sulcus in spatial attention. Brain 134, 1694-1709.

Glahn, D. C., Laird, A. R., EllisonWright, I., Thelen, S. M., Robinson, J. L., Lancaster, J. L., Bullmore, E., and Fox, P. T. (2008). Metaanalysis of gray matter anomalies in schizophrenia: application of anatomic likelihood estimation and network analysis. Biol. Psychiatry 64, 774-781.

Golay, L., Schnider, A., and Ptak, R. (2008). Cortical and subcortical anatomy of chronic spatial neglect following vascular damage. Behav. Brain Funct. 4:43. doi: 10.1186/1744-9081-4-43

Grimsen, C., Hildebrandt, H., and Fahle, M. (2008). Dissociation of egocentric and allocentric coding of space in visual search after right middle cerebral artery stroke. Neuropsychologia 46, 902-914.

Guariglia, C., and Antonucci, G. (1992). Personal and extrapersonal space - a case of neglect dissociation. Neuropsychologia 30, 1001-1009.

Halligan, P. W., Manning, L., and Marshall, J. C. (1990). Individual variation in line bisection: a study of 4 patients with right-hemisphere damage and normal controls. Neuropsychologia 28, 1043-1051.

Halligan, P. W., Marshall, J. C., and Wade, D. T. (1989). Visuospatial neglect: underlying factors and test sensitivity. Lancet 2, 908-911.

He, B. J., Snyder, A. Z., Vincent, J. L., Epstein, A., Shulman, G. L., and Corbetta, M. (2007). Breakdown of functional connectivity in frontoparietal networks underlies behavioral deficits in spatial neglect. Neuron 53, 905-918.

Heilman, K., Watson, R., and Valenstein, E. (1993). "Neglect and related disorders," in Clinical Neuropsychology, eds K. Heilman and E. Valenstein (New York, NY: Oxford University Press), 279-336.

Hillis, A. E. (2006). Neurobiology of unilateral spatial neglect. Neuroscientist 12, 153-163.

Hillis, A. E., Newhart, M., Heidler, J., Barker, P. B., Herskovits, E. H., and Degaonkar, M. (2005). Anatomy of spatial attention: insights from perfusion imaging and hemispatial neglect in acute stroke. J. Neurosci. $25,3161-3167$.

Husain, M., and Kennard, C. (1996). Visual neglect associated with frontal lobe infarction. J. Neurol. 243, 652-657.

Karnath, H. O., Berger, M. F., Kuker, W., and Rorden, C. (2004). The anatomy of spatial neglect based on voxelwise statistical analysis: a study of 140 patients. Cereb. Cortex 14, 1164-1172.

Karnath, H. O., Ferber, S., and Himmelbach, M. (2001). Spatia awareness is a function of the temporal not the posterior parietal lobe. Nature 411, 950-953.

Karnath, H. O., Himmelbach, M. and Kuker, W. (2003). The cortical substrate of visual extinction. Neuroreport 14, 437-442.

Karnath, H. O., Himmelbach, M., and Rorden, C. (2002). The subcortical anatomy of human spatial neglect: putamen, caudate nucleus and pulvinar. Brain 125, 350-360.

Karnath, H.-O., Rennig, J., Johannsen, L., and Rorden, C. (2011). The anatomy underlying acute versus chronic spatial neglect: a longitudinal study. Brain 134, 903-912.

Karnath, H. O., and Rorden, C. (in press). The anatomy of spatial neglect. Neuropsychologia. doi: 10.1016/ j.neuropsychologia.2011.06.027

Karnath, H. O., Rorden, C., and Ticini, L. F. (2009). Damage to white matter fiber tracts in acute spatial neglect. Cereb. Cortex 19, 2331-2337.

Kleinman, J. T., Newhart, M., Davis, C., Heidler-Gary, J., Gottesman, R. F, and Hillis, A. E. (2007). Right hemispatial neglect: frequency and characterization following acute left hemisphere stroke. Brain Cogn. 64 50-59.

Laird, A. R., Fox, P. M., Price, C. J., Glahn, D. C., Uecker, A. M., Lancaster, J. L., Turkeltaub, P. E., Kochunov, P., and Fox, P. T. (2005). ALE meta-analysis: controlling the false discovery rate and performing statistical contrasts. Hum. Brain Mapp. 25, 155-164.

Lancaster, J. L., Tordesillas-Gutierrez, D., Martinez, M., Salinas, F., Evans, A., Zilles, K., Mazziotta, J. C., and Fox, P. T. (2007). Bias between MNI and talairach coordinates analyzed using the ICBM-152 brain template. Hum. Brain Mapp. 28 1194-1205.

Leonards, U., Sunaert, S., Van Hecke, P., and Orban, G. A. (2000). Attention mechanisms in visual search - an fMRI study. J. Cogn. Neurosci. 12, 61-75.

Liu, G. T., Bolton, A. K., Price, B. H., and Weintraub, S. (1992). Dissociated perceptual-sensory and exploratory-motor neglect. $J$. Neurol. Neurosurg. Psychiatry 55, 701-706.
Mannan, S. K., Mort, D. J., Hodgson T. L., Driver, J., Kennard, C., and Husain, M. (2005). Revisiting previously searched locations in visual neglect: role of right parietal and frontal lesions in misjudging old locations as new. J. Cogn. Neurosci. 17, 340-354.

Marsh, E. B., and Hillis, A. E. (2008) Dissociation between egocentric and allocentric visuospatial and tactile neglect in acute stroke. Cortex 44, 1215-1220

Medina, J., Kannan, V., Pawlak, M. A., Kleinman, J. T., Newhart, M., Davis, C., Heidler-Gary, J. E., Herskovits, E. H., and Hillis, A. E. (2009). Neural substrates of visuospatial processing in distinct reference frames: evidence from unilateral spatial neglect. J. Cogn. Neurosci. 21, 2073-2084.

Mesulam, M. M. (1985). Principles of Behavioural Neurology. Philadelphia, PA: Davis.

Molenberghs, P., Gillebert, C. R. Peeters, R., and Vandenberghe, R. (2008). Convergence between lesion-symptom mapping and functional magnetic resonance imaging of spatially selective attention in the intact brain. J. Neurosci. 28, 3359-3373.

Molenberghs, P., Mesulam, M. M., Peeters, R., and Vandenberghe, R. R. C. (2007). Remapping attentional priorities: differential contribution of superior parietal lobule and intraparietal sulcus. Cereb. Cortex 17, 2703-2712.

Molenberghs, P., and Sale, M. V. (2011). Testing for spatial neglect with line bisection and target cancellation: are both tasks really unrelated? Plos One 6:23017. doi: 10.1371/journal.pone.0023017

Mori, S., Wakana, S., Nagae-Poetscher L. M., and van Zijl, P. C. M. (2005). MRI Atlas of Human White Matter. Amsterdam, Netherlands: Elsevier B.V.

Mort, D. J., Malhotra, P., Mannan, S. K., Rorden, C., Pambakian, A., Kennard, C., and Husain, M. (2003). The anatomy of visual neglect. Brain 126, 1986-1997.

Ogden, J. A. (1985). Anterior posterior interhemispheric differences in the loci of lesions producing visual hemineglect. Brain Cogn. 4, 59-75.

Ota, H., Fujii, T., Suzuki, K., Fukatsu, R., and Yamadori, A. (2001). Dissociation of body-centered and stimulus-centered representations in unilateral neglect. Neurology 57, 2064-2069.

Ptak, R., and Schnider, A. (2010). The dorsal attention network mediates orienting toward behaviorally 
relevant stimuli in spatial neglect. J. Neurosci. 30, 12557-12565.

Rengachary, J., He, B. J., Shulman, G. L., and Corbetta, M. (2011). A behavioral analysis of spatial neglect and its recovery after stroke. Front. Hum. Neurosci. 5:29. doi: 10.3389/fnhum.2011.00029

Rorden, C., Berger, M. F., and Karnath, H. O. (2006). Disturbed line bisection is associated with posterior brain lesions. Brain Res. 1080, $17-25$.

Rorden, C., Karnath, H. O., and Bonilha, L. (2007). Improving lesion-symptom mapping. J. Cogn. Neurosci. 19, 1081-1088.

Rotge, J.-Y., Langbour, N., Guehl, D., Bioulac, B., Jaafari, N., Allard, M., Aouizerate, B., and Burbaud, P. (2010). Gray matter alterations in obsessive-compulsive disorder: an anatomic likelihood estimation meta-analysis. Neuropsychopharmacology 35 , 686-691.

Schenkenberg, T., Bradford, D. C., and Ajax, E. T. (1980). Line bisection and unilateral visual neglect in patients with neurologic impairment. Neurology 30, 509-517.

Serences, J. T., and Yantis, S. (2007). Spatially selective representations of voluntary and stimulus-driven attentional priority in human occipital, parietal, and frontal cortex. Cereb. Cortex 17, 284-293.

Shallice, T., Mussoni, A., D’agostino, S., and Skrap, M. (2010). Right posterior cortical functions in a tumour patient series. Cortex 46, 1178-1188.

Stone, S. P., Halligan, P. W., and Greenwood, R. J. (1993). The incidence of neglect phenomena and related disorders in patients with an acute right or left-hemisphere stroke. Age Ageing 22, 46-52.

Stone, S. P., Halligan, P. W., Marshall, J. C., and Greenwood, R. J. (1998). Unilateral neglect: a common but heterogeneous syndrome. Neurology 50, 1902-1905.

Tatu, L., Moulin, T., Bogousslavsky, J., and Duvernoy, H. (2001). "Arterial territories of human brain," in Stroke Syndromes, eds J. Bogousslavsky and L. R. Caplan (Cambridge, MA: University Press), 375-404.

Thiebaut de Schotten, M., Urbanski, M., Duffau, H., Voue, E., Levy, R., Dubois, B., and Bartolomeo, P. (2005). Direct evidence for a parietal-frontal pathway subserving spatial awareness in humans. Science 309, 2226-2228.

Turkeltaub, P. E., Eden, G. F., Jones, K. M., and Zeffiro, T. A. (2002). Meta-analysis of the functional neuroanatomy of single-word reading: method and validation. Neuroimage $16,765-780$.

Urbanski, M., Thiebaut de Schotten, M., Rodrigo, S., Catani, M., Oppenheim, C., Touze, E., Chokron, S., Meder, J. F., Levy, R., Dubois, B., and Bartolomeo, P. (2008). Brain networks of spatial awareness: evidence from diffusion tensor imaging tractography. $J$. Neurol. Neurosurg. Psychiatry 79, 598-601.

Urbanski, M., Thiebaut de Schotten, M., Rodrigo, S., Oppenheim, C., Touze, E., Meder, J. F., Moreau, K., Loeper-Jeny, C., Dubois, B., and Bartolomeo, P. (2011). DTI-MR tractography of white matter damage in stroke patients with neglect. Exp. Brain Res. 208, 491-505.
Vallar, G., and Perani, D. (1986). The anatomy of unilateral neglect after right-hemipshere stroke lesions: a clinical CT-scan correlation study in man. Neuropsychologia 24, 609-622.

Vallar, G., Rusconi, M. L., Bignamini, L., Geminiani, G., and Perani, D. (1994). Anatomical correlates of visual and tactile extinction in humans: a clinical CT scan study. J. Neurol. Neurosurg. Psychiatry 57, 464-470.

Vandenberghe, R., and Gillebert, C. R. (2009). Parcellation of parietal cortex: convergence between lesionsymptom mapping and mapping of the intact functioning brain. Behav. Brain Res. 199, 171-182.

Vandenberghe, R., Molenberghs, P. and Gillebert, C. (in press). Spatial attention deficits in humans: the critical role of superior compared to inferior parietal lesions. Neuropsychologia. doi: 10.1016/ j.neuropsychologia.2011.12.016

Verdon, V., Schwartz, S., Lovblad, K. O., Hauert, C. A., and Vuilleumier, P. (2010). Neuroanatomy of hemispatial neglect and its functional components: a study using voxelbased lesion-symptom mapping. Brain 133, 880-894.

Vossel, S., Eschenbeck, P., Weiss, P. H., Weidner, R., Saliger, J., Karbe, H., and Fink, G. R. (2011). Visual extinction in relation to visuospatial neglect after right-hemispheric stroke: quantitative assessment and statistical lesion-symptom mapping. J. Neurol. Neurosurg. Psychiatry 82, 862-868.

Weintraub, S., and Mesulam, M. M. (1985). "Mental state assessment of young and elderly adults in behavioral neurology," in Principles of behavioral neurology, ed M. M. Mesulam (Philadelphia, PA: Davis Company), 71-123.

Wilson, B., Cockburn, J., and Halligan, P. W. (1987). Behavioural Inattention Test. Thames Valley: Bury St. Edmunds.

Woldorff, M. G., Hazlett, C. J., Fichtenholtz, H. M., Weissman, D. H., Dale, A. M., and Song, A. W. (2004). Functional parcellation of attentional control regions of the brain. J. Cogn. Neurosci. 16, 149-165.

Zopf, R., Berger, M. F., Klose, U., and Karnath, H.-O. (2009). Perfusion imaging of the right perisylvian neural network in acute spatial neglect. Front. Hum. Neurosci. 3:15. doi: 10.3389/neuro.09.015.2009

Conflict of Interest Statement: The authors declare that the research was conducted in the absence of any commercial or financial relationships that could be construed as a potential conflict of interest.

Received: 22 January 2012; accepted: 20 March 2012; published online: 10 April 2012.

Citation: Molenberghs $P$, Sale $M V$ and Mattingley JB (2012) Is there a critical lesion site for unilateral spatial neglect? A meta-analysis using activation likelihood estimation. Front. Hum. Neurosci. 6:78. doi: 10.3389/fnhum.2012.00078 Copyright (c) 2012 Molenberghs, Sale and Mattingley. This is an open-access article distributed under the terms of the Creative Commons Attribution Non Commercial License, which permits noncommercial use, distribution, and reproduction in other forums, provided the original authors and source are credited. 